The description is prepared from a male collected by my friend $\mathrm{Mr}$. A. C. Jensen-Haarup, to whom I dedicate the species. It was collected at Guden River (Aastedbro) 16. V. 08, Denmark.

I herewith beg Mr. Eaton to receive my best thanks for his valuable advice and hints.

\title{
Beiträge zur Kenntnis der Cerambycidenfauna der deutschen Kolonien Afrikas. I. (Col.)
}

Von E. Hintz, Berlin.

Dem Missionsmuseum zu Steyl und besonders dem rührigen Ordner desselben, Herrn Br. Berchmans I. verdanke ich eine Anzahl von Cerambyciden aus Togo, von denen sich mehrere Arten als neu erwiesen haben.

Die neuen Arten dieser Togo-Ausbeute und einige neue Arten meiner Sammlung aus Deutsch-Ostafrika werden nachstehend beschrieben.

Die Typen der neuen Arten aus Togo befinden sich im Missionsmuseum zu Steyl und in meiner Sammlung, die Typen der neuen Arten aus Ostafrika in meiner Sammlung.

$$
\text { Aulacopus orientalis n. sp. }
$$

Niger vel nigro-cyaneus, elytris interdum purpureis, opacus; capite fortiter punctato-granulato, fronte medio ante tuberculos antenniferos semicirculariter impresso, vertice impresso, postice canaliculato; prothorace fortiter densissime granulose punctato, plus minusve canaliculato, interdum solomodo basi breviter impresso, dorso parum nitido, lateribus parum denticulatis, angulis anticis brevissime, angulis posticis longius spinosis; scutello plano, nitido, fortiter punctato; elytris coriaceis, circa scutellum elevatis et fortiter scabrosis, 9-costatis; antennis medium elytrorum paulo superantibus, nitidis; subtus undique aequaliter punctato. Long. $20-24 \mathrm{~mm}$. -3 Ex., Lindi.

$$
\text { Xystrocera togonica n. sp. }
$$

Capite, prothorace elytrisque viridis, subtus brunneo-ferruginea; capite rugoso-punctato; prothorace subtiliter densissime punctato, nitido, elytrorum latitudine perparum breviore quam latiore, dorso W-impresso; elytris densissime rugoso-punctatis, ob- 
solete tricostatis; antennis $\left(\sigma^{7}\right)$ corpore fere duplo longioribus, piceis, articulo primo brunneo, articulis 4 anticis fortiter rugose punctatis; pectore plus minusve flavo-tomentoso; pedibus piceis, femoribus furrugineo-clavatis. - Long. 16-28 mm. - $4 \sigma^{7} \sigma^{7}$ Kpandu (Togo). 2 Stücke im Missionsmuseum zu Steyl und 2 Stücke in meiner Sammlung.

Das + wird sich vermutlich durch kürzere Fühler und ein kleineres Halsschild von dem beschriebenen $\sigma^{7}$ unterscheiden.

Die Art ist am ähnlichsten der $X$. lateralis Chevr.

$$
\text { Prosopocera grisea n. sp. }
$$

Grisea, supra tomento griseo obscure brunneo-variegata, infra tomento brunneo-grisescente vestita; capite sparsim nigro-granulato, linea media longitudinali, lineis transversis semicircularibus inter tuberculos antenniferos et pone clipeum nigris; prothorace transverso cylindrico, sparsim nigro-punctato, utrinque spina submediana brevi acuta armato; elytris ad humeros fortiter, deinde subtilius nigro-granulatis, apice conjunctim rotundatis, angulo suturali brevissime dentato, maculis duabus rotundis atrotomentosis ante medium ornatis, altera parva dorsali, altera majore ad marginem pone humeros sita; corpore infra sparsissime subtiliterque nigro-punctato; tibiis tarsisque flavescentibus, antennis sanguineis, flavo-pubescentibus. - Long. $18 \mathrm{~mm}$ - - $1 \mathrm{Ex}$. aus Palime (Togo) in meiner Sammlung. Weitere Stücke im Missionsmuseum zu Steyl.

\section{Prosopocera Janseni n. sp.}

Pr. Ertli Auriv. affinis. Sanguinea, supra argenteo-tomentosa, infra et capite, prothorace (antice et postice), scutello et elytrorum basi flavo-tomentosa; capite maris cornu valido curvato armato, longitudinaliter sulcato, inter oculos semicirculariter impresso, cornu, frontis lateribus et juxta oculos nigro-granulato; prothorace breviore quam latiore, utrinque spina brevi mediana armato, basi apiceque transversim biimpresso, dorso fere plano, nigro ruguloso; scutello rotundato; elytris sensim apicem versus distincte angustatis, nigro-punctatis, humeris fortiter nigro-granulatis, apice singulariter rotundatis, inermibus, macula rotunda dorsali ante medium, interdum plagaque laterali pone humeros atrotomentosa ornatis; corpore infra impunctato; pedibus antennisque griseo-tomentosis; antennis articulis $1^{0} \quad 2^{0} 3^{0}$ crassis, articulis $4-8$ infra ciliatis. - Long. 26-31 mm. - 2 Ex. Palime et Kpandu (Togo). Dem Andenken des Hochw. Herrn P. Generalsuperior J a n s s e n gewidmet. 


\section{Prosopocera assimilis n. sp.}

Pr. marmorata Gah. affinis. Sanguinea, supra albido-tomentosa et marmorata, infra et capite, prothorace, scutello et elytrorum basi flavo-tomentosa; capite maris cornu brevi, apice bifido, armato, longitudinaliter nigro-carinato, frontis lateribus nigrogranulatis, inter oculos impresso; prothorace subquadrato, utrinque dente obtuso armato, basi apiceque bisulcato; elytris apice conjunctim rotundatis, ad humeros fortiter, deinde sparsim apicem versus obsolete punctatis, haud granulatis, utrinque tribus maculis albido- vel flavescente-tomentosis, macula rotunda ad marginem pone humeros, plaga fere in medio plagaque pone medium; corpore infra impunctato; antennis griseo-tomentosis, articulis 5-10 prope apicem brunneo-annulatis, articulis (o $) 1-3^{0}$ crassis. Long. 19-23 mm. - $1 \sigma^{7}$ Uganda (Brit.-Ostafrika), 2 q $q$ Lindi (Deutsch-Ostafrika).

\section{Prosopocera minor n. sp.}

Sanguinea, albido- vel griseo-tomentosa; capite maris cornu curvato, interdum brevi, apice bifido, armato, vertice impresso, longitudinaliter nigro-carinato, frontis lateribus granulato; prothorace quadrato, lateribus inermi, basi apiceque bisulcato; elytris apice coniunctim rotundatis, ad humeros fortiter, deinde sparsim sed fere aequaliter punctatis, haud granulatis; corpore infra impunctato; antennis griseo-tomentosis, articulis $3^{0}$ et $4^{0}$ maris perparum incrassatis. - Long. $14-18 \mathrm{~mm}$. - $2 \sigma^{\top} \sigma^{\top}$, 3 우 우 Lindi (Deutsch-Ostafrika.)

\section{Tragocephala Berchmansi n. sp.}

Nigra, flavo-fasciata; capite flavo, utrinque stria ante oculos, linea inter oculos et plaga ad basin nigris; prothorace flavo, fascia mediana late nigra, dorso flavo-maculato, in medio fortiter spinoso; scutellum flavum; elytris fascia basali lata communi lateraque versus dilatata, basin circum scutellum haud attingente, humeris maculaque minuta pone humeros exceptis, fascia mediana lata, maculis duabus minutis singulis, fascia anteapicali anguloque apicali flavis; corpore infra flavo-tomentoso, impunctato; antennis nigris, articulis apice perparum albidis; pedibus griseo-, infra flavo-tomentosis. - Long. 19-20 mm. - 2 Ex. Kpandu (Togo).

\section{Tragocephala similis n. sp.}

Tr. velutinae Thoms. affinis. Nigra, velutina, flavo-fasciata; capite 3-maculata, macula latissima mediana et duabus maculis obliquis conjunctis pone oculos; prothorace lateribus longitudi- 
naliter late fasciato, pone medium breve spinoso; elytris fascia basali lata communi basin circum scutellum haud attingente, humeris exceptis, fascia mediana - interdum confluentibus coarctata, parum obliquata maculaque anteapicali cum maculis duabus parvis confluente flavis, angulo apicali et duarum macularum interiore albidis; corpore infra albido-viride-tomentoso, mesosterno lateraliter nigro, abdominis segmenta in medio et in lateribus nigro-maculata; antennis piceis. - Long. 18-20 mm. 2 Ex. Kpandu (Togo).

\section{Tragocephala Probi n. sp.}

Nigra, viride-fasciata; capite 5-maculata, macula frontali, utrinque maculis duabus ante et pone oculos; lateribus prothoracis longitudinaliter late fasciatis, pone medium breve nigrospinosis; elytris fascia basali lata communi lateraque versus dilatata, basin elytrorum haud attingente, humeris interrupta, fascia mediana angusta haud obliquata, suture apicem versus reducta, utrinque maculis duabus minutis singulis macula singula ovali anteapicali anguloque apicali viridibus; corpore infra viridetomentoso, pectoris lateribus nigro-plagiatis; abdomine in medio et lateribus nigro-plagiato, segmento ultimo utrinque ad basin nigro. - Long. 18-20 mm. - 3 Ex. Kpandu (Togo).

Dem Andenken des ehrw. Herrn Br. Probus gewidmet.

$$
\text { Tragocephala pretiosa n. sp. }
$$

Nigra, flavo-fasciata; capite flavo, stria juxta oculos, linea inter oculos et plaga ad basin nigris; prothorace flavo, fascia mediana late nigra, pone medium fortiter spinoso; scutellum nigrum; elytris fasciis tribus flavis ornatis, fascia basali lata basin haud attingente, fascia mediana lata parum obliquata fasciaque anteapicali suture interrupta; corpore infra flavo-tomentoso, pectoris lateribus nigro-plagiatis; abdomine in medio et lateribus nigro-plagiato; antennis nigris; pedibus nigris, femoribus basi flavo-tomentosis. - Long. $23 \mathrm{~mm}$. - 2 Ex. Lutindi et Nguelo (Usambara).

\section{Tragocephala nyassica n. sp.}

Nigra, flavo-plagiata; capite prothoraceque nigris; scutello nigro; elytris nigris, pone humeros ad marginem utrinque flavoplagiatis, parum pone medium fascia transversa parum coarctata suture interrupta et utrinque plaga apicali magna ornatis, interdum inter fasciam et plagam apicalem utrinque puncto minuto albo signatis; corpore infra griseo-pubescente, abdomine utrinque 
flavo-maculato, segmento apicali nigro; antennis griseo-pubescentibus. - Long. 21-27 mm. - 4 Ex. Nyassa (Deutsch-Ostafrika).

\section{Phantasis Thoms.}

Die Arten dieses Genus sind einander sehr ähnlich und weisen nur geringe Unterscheidungsmerkmale auf, so dafs nach der Beschreibung nur schwer die Zugehörigkeit der Arten festzustellen ist. Bei einer Bearbeitung dieses Genus wird sich zweifellos herausstellen, dafs eine Anzahl von Artnamen eingezogen werden mufs.

Bei der Untersuchung einer grofsen Anzahl von Stücken hat sich die Ausbildung der Flügeldeckenenden als gutes Unterscheidungsmerkmal erwiesen. Mit Hülfe dieses Merkmals dürfte sich eine Bestimmungstabelle der Phantasisarten ohne erhebliche Schwierigkeiten aufstellen lassen.

Die nachstehend beschriebenen Arten haben sich nach Vergleichung mit den Kolbeschen Typen als neu erwiesen.

\section{Phantasis spinosa n. s.}

Brunnea, fusco-tomentosa ; capite prothoraceque fortiter punctatis, nigro-setosis, hoc haud breviore quam latiore, dorso medio biseriatim octo-nodoso, nodis 2 anticis curvatis, nodis 2 posticis minime distinctis; elytris tricostatis, costis dentatis, interstitiis granulatis, granulis tomentosis, apice elytrorum utrinque sinuato, angulo suturali bispinoso, angulo laterali plus minusve dentiformi; pedibus fuliginosis, genubus albidis; antennis robustis, hirtis; $\sigma^{7}$ palporum articulo ultimo late securiformi, $q$ eodem angustato, subsecuriformi, fere duplo longiore quam latiore. - Long. 22-32 mm. - 5 Ex. in meiner Sammlung aus Peramiho (DeutschOstafrika).

Die Behaarung und Form der Fühler weist die vorliegende Art in die Verwandtschaft von $P h$. phantoma Kolbe, während die Tomentirung der ganzen Flügeldecken sie in die Verwandtschaft von gigantea Guér. bringt.

Ein Merkmal der vorliegenden Art bilden die verhältnismälsig dünnen Beine, die bei keiner der mir bekannten Arten ähnlich dünn sind. Die Rippen auf den Flügeldecken sind verschieden stark gezähnt, bei den $\sigma^{\top} \sigma^{\top}$ jedenfalls erheblich stärker als bei den vorliegenden $q$ ㅇ․

\section{Phantasis meridionalis n. sp.}

Nigro-fusca, fulvo-tomentosa; capite prothoraceque sparsim punctatis, illo antice setis nigris erectis vestito, hoc haud breviore quam latiore, dorso medio biseriatim octo-nodoso, nodis anticis curvatis, posticis parum minus distinctis quam illis; elytris tri- 
costatis, costis alternatim tuberculatis, interstitiis granulatis, granulis nitidis, apice elytrorum utrinque sinuato, angulo suturali paulo producto, laterali producto; pedibus umbrinis, femorum apice pallido; antennis robustis, parum setosis; $\sigma$ palporum articulo ultimo late securiformi. - Long. $31 \mathrm{~mm}$. - 1 o Karibib (Deutsch-Südwestafrika) in meiner Sammlung, 2 weitere Stücke in der Sammlung des Herrn Hauptmann Mos er.

In der Struktur der Flügeldecken der $P$. phantoma Kolbe am nächsten, die Fühler sind jedoch weit weniger behaart. Die Art fällt auf durch die stark vorstehenden Fühlergrubenwände, die bei keiner der übrigen Arten so hervortreten.

\section{Phantasis manowensis n. sp.}

Atro-fusca, griseo-variegata; capite antice rude punctato, setis nigris erectis vestito; prothorace breviore quam latiore, foveolato, tuberoso, tuberibus biseriatis, medianis compressis, duplicatis, crassis, carinulis duabus anticis haud curvatis, crassis, carinulis duabus posticis exiguis; elytris tricostatis, costis paulo elevatis, interruptis, interstitiis foveolatis et granulatis, apice utriusque elytris paulo sinuato, angulis lateralibus parum productis, angulo suturali haud producto; pedibus umbrinis; antennis gracilibus, parum setosis; $q$ palporum articulo ultimo angustato, apice obtuso. - Long. $26 \mathrm{~mm}$. - 1 Ex. Manow (Deutsch-Ostafrika) in meiner Sammlung, von der Firma Dr. O. Sta u dinger und $\mathrm{B}$ a $\mathrm{ng}-\mathrm{H}$ a a s freundlichst abgetreten.

Die Art gehört in die Verwandtschaft von $P$. petrificata Kolbe und ist leicht $\mathrm{zu}$ erkennen an dem Apikalwinkel der Flügeldecken und an den stark aufgetriebenen oben platten und mit einem Querspalt versehenen Mittelbeulen des Halsschildes.

\section{Phantasis lukuledensis n. sp.}

Umbrina, griseo-maculosa, femorum apice pallido; capite antice sparsim, vertice crebre fortiter punctato, tuberculo utriusque iuxtaoculari indistincto, tuberculis antenniferis modice elevatis, subacutis, fovea media impunctata; prothorace tuberoso, foveolato, rugoso, carinulis duabus anticis curvatis, tuberibus duobus mediis duplicatis, spatio mediano binodulo et foveolato, tuberculis 2 posticis exiguis; elytris scabrosis, tricostatis, costis elevatis vel dupliciter vel alternatim tuberculatis, costarum tuberculis acutis, erectis, indistincte continuis, interstitiis foveolatis et granulatis, granulis nitidis, elytris apice coniunctim sinuatis, angulo exteriore dentiformi; or palporum articulo ultimo late securiformi, $q$ eodem angustato, dimidio longiore quam latiore; segmento abdominali ultimo postice haud sinuato. - Long. 23-25 mm. - 25 Ex. Lukuledi. 
Mit Ph. ominosa Kolbe verwandt, hauptsächlich unterschieden durch die andere Ausbildung des Apikalwinkels der Flügeldecken.

Ferner sind die Rippen auf den Flügeldecken mehr in einzelne Zacken aufgelöst, besonders bei den $\sigma^{\top} \sigma^{\top}$, so dafs sie aus Reihen von spitzen nach hinten gerichteten Zähnen bestehen.

\section{Phantasis grisea n. sp.}

Griseo-tomentosa; pectore pedibusque griseo-fuscis, abdomine brunneo, utrinque triseriatim maculoso, maculis duarum serierum exteriorum albidis; capite nigro-setoso, vage, fronte dense, fortiter punctato, vertice distincte nigro-carinulato, impresso, infra oculos punctato; prothorace haud breviore quam latiore, punctato, dorso medio biseriatim octo-tuberculoso, carinulis 2 anticis plus minusve rectis, tuberibus medianis compressis, duplicatis, 2 posticis parvis; elytris tricostatis, costis dentatis, interstitiis foveolatis, haud granulatis, apice elytrorum utrinque sinuato, angulo suturali perparum producto, angulo laterali dentiformi ; pedibus fuliginosis, genubus albidis; antennis gracilibus, hirtis; $\sigma^{7}$ palporum articulo ultimo subsecuriformi, $q$ eodem angustato duplo longiore quam latiore. - Long. 19-27 mm. - 3 Ex. Mahenge (Deutsch-Ostafrika) in meiner Sammlung, von der Firma Dr. O. St a u dinger und $\mathrm{Bang}-\mathrm{Ha}$ a freundlichst abgetreten. Die ganze Oberseite ist hellgrau tomentiert. Die Rippen auf den Flügeldecken sind gezähnt. Die Zwischenräume zwischen den Rippen sind stark grubig punktiert, ohne Granulierung.

\section{Eine auffallende neue Form von Anaglyptus mysticus L.:} Bequaerti ab. nov. (Col.)

\section{Von Prof. J. Roubal, Prag-Vinohrady.}

Die 3 Tomentbinden in der Mitte der Flügeldecken fliefsen zu einem grofsen Tomentfeld zusammen, blofs zwischen der ehemaligen ersten und der zweiten Binde ist ein sehr schwaches Spatium angedeutet. Während bei dem Typ die Tarsen rötlichgelb sind, sind bei der ab. Bequaerti die 2 ersten Tarsenglieder ganz dunkel, die andern rotbraun. Die Behaarung der Episternen von Mittel- und Hinterbrust ist weifs, bei der Stammform gelblichweifs. Ganglbauer sagt auch in Best.-Tab. Ceramb., pg. 738 : "die Episternen . . . weils behaart". Diese sehr schöne Aberration erhielt ich von Herrn Dr. Jos. B equa ert (Aalst, Belgien), dem ich sie auch widme, mit einigen gemeinen undeterminierten Coleopteren aus Belgien. Der Käfer ist bezettelt: Aalst 2. VII. 1908; Dr. J. Bequa ert. - (Coll. Rouba l.) 


\section{$2 \mathrm{BHL}$ Biodiversity Heritage Library}

Hintz, E . 1909. "Beiträge zur Kenntnis der Cerambycidenfauna der deutschen Kolonien Afrikas. I. (Col.)." Deutsche entomologische Zeitschrift 1909(4), 556-562. https://doi.org/10.1002/mmnd.48019090413.

View This Item Online: https://www.biodiversitylibrary.org/item/103377

DOI: https://doi.org/10.1002/mmnd.48019090413

Permalink: https://www.biodiversitylibrary.org/partpdf/235972

\section{Holding Institution}

Harvard University, Museum of Comparative Zoology, Ernst Mayr Library

\section{Sponsored by}

Biodiversity Heritage Library

\section{Copyright \& Reuse}

Copyright Status: Public domain. The BHL considers that this work is no longer under copyright protection.

This document was created from content at the Biodiversity Heritage Library, the world's largest open access digital library for biodiversity literature and archives. Visit BHL at https://www.biodiversitylibrary.org. 